\title{
A SUM CONNECTED WITH QUADRATIC RESIDUES
}

\author{
L. CARLITZ
}

1. Let $p$ be a prime $>2$ and $m$ an arbitrary positive integer; define

$$
S_{m}=\sum_{r=0}^{m}(-1)^{m-r}\left(\frac{r}{p}\right)\left(\begin{array}{c}
m \\
r
\end{array}\right)
$$

where $(r / p)$ is the Legendre symbol. We consider the problem of finding the highest power of $p$ dividing $S_{m}$. A little more generally, if we put

$$
S_{m}(a)=\sum_{r=0}^{m}(-1)^{m-r}\left(\frac{r+a}{p}\right)\left(\begin{array}{c}
m \\
r
\end{array}\right),
$$

where $a$ is an arbitrary integer, we seek the highest power of $p$ dividing $S_{m}(a)$. Clearly $S_{m}=S_{m}(0)$, and $S_{m}(a)=S_{m}(b)$ when $a \equiv b(\bmod p)$.

In the first place it follows from (1.2) that $S_{m}(a)$ satisfies the recurrence

$$
S_{m+1}(a)=\Delta S_{m}(a)=S_{m}(a+1)-S_{m}(a),
$$

where it is understood that $\Delta$ applies only to $a$. Repeated application of (1.3) gives

$$
S_{m+r}(a)=\Delta^{r} S_{m}(a)=\sum_{s=0}^{r}(-1)^{r-s}\left(\begin{array}{c}
r \\
s
\end{array}\right) S_{m}(a+s) .
$$

We may also write (1.3) in the form

$$
S_{m}(a+1)=S_{m+1}(a)+S_{m}(a)
$$

which implies

$$
S_{m}(a+r)=\sum_{s=0}^{r}\left(\begin{array}{l}
r \\
s
\end{array}\right) S_{m+s}(a) .
$$

In particular for $r=p,(1.6)$ becomes

$$
\sum_{s=1}^{p}\left(\begin{array}{c}
p \\
s
\end{array}\right) S_{m+s}(a)=0 .
$$

2. It follows from

Received August 8, 1955. 


$$
\left(\frac{r}{p}\right) \equiv r^{(p-1) / 2} \quad(\bmod p)
$$

that $\quad\left(\frac{r}{p}\right) \equiv r^{p^{n}(p-1) / 2} \quad\left(\bmod p^{n+1}\right)$

for arbitrary $n \geqslant 0$. Consequently (1.2) becomes

$$
S_{m}(a)=\sum_{r=0}^{m}(-1)^{m-r}\left(\begin{array}{c}
m \\
r
\end{array}\right)(r+a)^{p^{n}(p-1) / 2} \quad\left(\bmod p^{n+1}\right) .
$$

We recall that for arbitrary positive $k$

$$
\frac{1}{m !} \sum_{r=0}^{m}(-1)^{m-r}\left(\begin{array}{c}
m \\
r
\end{array}\right)(r+a)^{k}
$$

is an integer (for $a=0,(2.2)$ is a Stirling number of the second kind). If now $E_{p}(m)$ denotes the highest power of $p$ dividing $m !$, it is clear from (2.1) that

$$
S_{m}(a) \equiv 0 \quad\left(\bmod p^{E_{p}(m)}\right) .
$$

In view of the definition of $E_{p}(m),(2.3)$ may be restated in the following way: $S_{m}(a) / m$ ! is integral $(\bmod p)$.

3. It may be possible to improve (2.3). We make use of the following familiar formula for Gauss sums (see for example [2, Th. 215]) :

$$
\sum_{s=1}^{p-1}\left(\frac{s}{p}\right) \varepsilon^{r s}=\left(\frac{r}{p}\right) G_{p} \quad\left(\varepsilon=e^{2 \pi i / p}\right)
$$

where

$$
G_{p}=\sum_{s=1}^{p-1}\left(\frac{s}{p}\right) e^{s}= \begin{cases}p^{1 / 2} & (p \equiv 1(\bmod 4)) \\ i p^{1 / 2} & (p \equiv 3(\bmod 4))\end{cases}
$$

Note that (3.1) is valid for all $r$. It follows that

$$
G_{p} S_{m}(a)=\sum_{r=0}^{m}(-1)^{m-r} \sum_{s=1}^{p-1}\left(\frac{s}{p}\right) \varepsilon^{(r+a) s}
$$

so that

$$
G_{p} S_{m}(a)=\sum_{s=1}^{p-1}\left(\frac{s}{p}\right) \varepsilon^{a s}\left(\varepsilon^{s}-1\right)^{m}
$$

Clearly (3.3) implies

$$
G_{p} S_{m}(a) \equiv 0 \quad\left(\bmod (\varepsilon-1)^{m}\right)
$$


where we are now operating in the cyclotomic field $k(\varepsilon)$. Since in this field we have

$$
(p)=(\varepsilon-1)^{p-1},
$$

(3.2) and (3.4) yield

$$
S_{m}(a) \equiv 0 \quad\left(\bmod (\varepsilon-1)^{m-(p-1) / 2}\right) .
$$

Define the integer $h$ by means of

$$
(h-1)(p-1)<m-\frac{1}{2}(p-1) \leqq h(p-1) .
$$

Since $S_{m}(a)$ is a rational integer, it follows from (3.6) and (3.7) that

$$
S_{m}(a) \equiv 0 \quad\left(\bmod p^{h}\right)
$$

which again is valid for all $a$.

We recall that

$$
E_{p}(m)=\left[\frac{m}{p}\right]+\left[\frac{m}{p^{2}}\right]+\ldots<\frac{m}{p-1} ;
$$

hence using (3.7) we may verify that $h \supseteq E_{p}(m)$ so that (3.8) implies (2.3). In particular for

$$
\frac{1}{2}(p-1)<m \leqq p-1,
$$

$h=1$ while $E_{p}(m)=0$. The difference $h-E_{p}(m)$ may indeed be arbitrarily large; for example if

$$
p^{k}-1-\frac{1}{2}(p-1)<m \leqq p^{k}-1
$$

we find that

$$
E_{p}(m)=\frac{p^{k}-1}{p-1}-k, \quad h=\frac{p^{k}-1}{p-1}
$$

so that $h-E_{p}(m)=k$.

4. Returning to (3.3) we consider the particular case

$$
m-\frac{1}{2}(p-1)=h(p-1)
$$


for such $m$ the value of $h$ computed by means of (3.7) will coincide with the. value of $h$ in (4.1). Now (3.3) implies

$$
(\varepsilon-1)^{-m} G_{p} S_{m}(a)=\sum_{s=1}^{p-1}\left(\frac{s}{p}\right) \varepsilon^{a s}\left(\frac{\varepsilon^{s}-1}{\varepsilon-1}\right)^{m} .
$$

We shall compute the residue of the right member $(\bmod \varepsilon-1)$. Since

$$
\varepsilon^{a s} \equiv 1, \quad \frac{\varepsilon^{s}-1}{\varepsilon-1} \equiv s,
$$

it is evident that (4.2) becomes

$$
\begin{aligned}
(\varepsilon-1)^{-m} G_{p} S_{m}(a) \equiv \sum_{s=1}^{p-1}\left(\frac{s}{p}\right) s^{m} & \equiv \sum_{s=1}^{p-1}\left(\begin{array}{c}
s \\
p
\end{array}\right) s^{(p-1) / 2} \\
& =\sum_{s=1}^{p-1} s^{p-1} \equiv-1 \quad(\bmod \varepsilon-1) .
\end{aligned}
$$

Next we replace $(3.5)$ by the more exact statement

$$
p \equiv(\varepsilon-1)^{p-1} \quad\left(\bmod (\varepsilon-1)^{p}\right),
$$

which is easily proved. Also if $p=2 k+1$, the identity (see for example [3, p. 176])

$$
\sum_{0}^{p-1} \varepsilon^{s(s+1)}=\prod_{1}^{k}\left(1-\varepsilon^{-2(2 s-1)}\right)
$$

implies

$$
G_{p}=\sum_{0}^{p-1} \varepsilon^{s^{2}} \equiv(-1)^{k}(\varepsilon-1)^{k} k ! \quad\left(\bmod (\varepsilon-1)^{k+1}\right)
$$

Using (4.4) and (4.5), (4.3) becomes

$$
p^{-h} S_{m}(a) \equiv-(-1)^{k} / k ! \quad(\bmod p) .
$$

Hence for $m$ satisfying (4.1) the exponent $h$ furnishes the highest power of $p$ dividing $S_{m}(a)$ and the residue of $p^{-h} S_{m}(a)$ satisfies (4.6). Note also that the right member of (4.6) is independent of $a$.

5. When $m$ does not satisfy (4.1) it is more difficult to simplify the right member of (4.2). Let

$$
(h-1)(p-1)<m-\frac{1}{2}(p-1)<h(p-1) ;
$$

it is convenient to put 


$$
m+l=h(p-1)+\frac{1}{2}(p-1) \quad(1 \leqq l \leqq p-2) .
$$

Thus it is clear from (3.8) that the right member of (4.2) is divisible by $(\varepsilon-1)^{l}$ and we have

$$
(\varepsilon-1)^{-h(p-1)} G_{p} S_{m}(a) \equiv(\varepsilon-1)^{-l} \sum_{s=1}^{p-1}\left(\frac{s}{p}\right) \varepsilon^{a s}\left(\frac{\varepsilon^{s}-1}{\varepsilon-1}\right)^{m} \quad(\bmod \varepsilon-1) .
$$

We accordingly seek the residue of

$$
T_{m}(a)=\sum_{s=1}^{p-1}\left(\begin{array}{c}
s \\
p
\end{array}\right) \varepsilon^{a s}\left(\frac{\varepsilon^{s}-1}{\varepsilon-1}\right)^{m}\left(\bmod (\varepsilon-1)^{l+1}\right) .
$$

Clearly we may put

$$
T_{m}(a) \equiv A_{0}+A_{1}(\varepsilon-1)+\ldots+A_{l}(\varepsilon-1)^{l},
$$

where the $A$ 's are rational integers; it follows from $(3.8)$ that $A_{0} \equiv \ldots \equiv A_{l-1}$ $\equiv 0(\bmod p)$ and may therefore be ignored. Thus in the expansion of the right member of (5.4) we need only retain the term in $(\varepsilon-1)^{l}$. Now we have

$$
\left(\begin{array}{c}
(1+x)^{s}-1 \\
x
\end{array}\right)^{m}=x^{-m} \sum_{r=0}^{m}(-1)^{m-r}\left(\begin{array}{c}
m \\
r
\end{array}\right)(1+s)^{r s},
$$

so that

$$
\begin{aligned}
(1+x)^{a s}\left(\begin{array}{c}
(1+x)^{s}-1 \\
x
\end{array}\right)^{m} & =x^{-m} \sum_{r=0}^{m}(-1)^{m-r}\left(\begin{array}{c}
m \\
r
\end{array}\right)(1+x)^{(a+r) s} \\
& =\sum_{r=0}^{m}(-1)^{m-r}\left(\begin{array}{c}
m \\
r
\end{array}\right) \sum_{t=0}^{(a+r) s}\left(\begin{array}{c}
(a+r) s \\
t
\end{array}\right) x^{t-m}
\end{aligned}
$$

Hence by the above remark we get

$$
(\varepsilon-1)^{-l} T_{m}(a) \equiv \sum_{s=1}^{p-1}\left(\frac{s}{p}\right) \sum_{r=0}^{m}(-1)^{m-r}\left(\begin{array}{c}
m \\
r
\end{array}\right)\left(\begin{array}{c}
(a+r) s \\
m+l
\end{array}\right) \quad(\bmod \varepsilon-1) .
$$

To further simplify this result note that the inner sum in the right member is the $m$-th difference of a polynomial in $a$ of degree $m+l$; thus only terms of degree $\geqslant m$ make any contribution. Now for a term of degree $t$, where $m \leqq t \leqq m+l$, we get

$$
\sum_{s=1}^{p-1}\left(\frac{s}{p}\right) s^{t} \equiv \sum_{s=1}^{p-1} s^{(p-1) / 2+t}
$$

and in view of $(5.2)$ this sum vanishes $(\bmod p)$ unless $t=m+l$ in which case the sum $\equiv-1$. Thus $(5.5)$ becomes 


$$
(\varepsilon-1)^{-l} T_{m}(a) \equiv-\frac{1}{(m+l) !} \sum_{r=0}^{m}(-1)^{m-r}\left(\begin{array}{c}
m \\
r
\end{array}\right)(a+r)^{m+l} \quad(\bmod \varepsilon-1) .
$$

Finally as in the proof of (4.6), we may simplify the left member of (5.3). Thus using (5.4) and (5.6) we obtain

$$
p^{-h} S_{m}(a) \equiv-\frac{(-1)^{k}}{k !} \frac{1}{(m+l) !} \sum_{r=0}^{m}(-1)^{m-r}\left(\begin{array}{c}
m \\
r
\end{array}\right)(a+r)^{m+l} \quad(\bmod p),
$$

where $p=2 k+1$ and $h$ and $l$ are defined by (5.1) and (5.2). When $m$ satisfies (4.1) it is easily verified that (5.7) reduces to (4.6). Thus (5.7) holds for all $m$. We may therefore state the following

THEOREM. Let $p=2 k+1$ be a prime, $a$ an arbitrary integer and $m$ a positive integer; define $h$ and $l$ by means of

$$
(h-1)(p-1)<m-\frac{1}{2}(p-1) \leqq h(p-1), \quad m+l=h(p-1)+\frac{1}{2}(p-1) .
$$

Then $S_{m}(a)$ satisfies (5.7). In particular when $l=0$, (5.7) reduces to

$$
p^{-h} S_{m}(a) \equiv-\frac{(-1)^{k}}{k !} \quad(\bmod p) \quad(l=0)
$$

Comparison of (5.7) with (2.1) leads to a rather curious congruence.

It should be remarked that the right member of (5.7) may be divisible by $p$; thus we have not in all cases determined the highest power of $p$ dividing $S_{m}(a)$. However when $m=h(p-1)+(p-1) / 2, h$ is the correct exponent.

For small values of $l$, the right member of (5.7) can be reduced further using known properties of Stirling numbers of the second kind (see for example $[1, \S 58]$ and $[4])$ :

$$
A_{m+l, m}=\frac{1}{m !} \sum_{r=0}^{m}(-1)^{m-r}\left(\begin{array}{c}
m \\
r
\end{array}\right) r^{m+l} .
$$

We have in particular

$$
\begin{aligned}
& A_{m+1, m}=\frac{1}{2} m(m+1), \\
& A_{n+2, m}=\frac{1}{24} m(m+1)(m+2)(3 m+1), \\
& A_{m+3, m}=\frac{1}{48} m^{2}(m+1)^{2}(m+2)(m+3) .
\end{aligned}
$$

On the other hand it is clear that (5.7) can be rewritten as 


$$
p^{-h} S_{m}(a)=-\frac{(-1)^{k}}{k !} \frac{1}{(m+1) \ldots(m+l)} \sum_{t=m}^{m+l}\left(\begin{array}{c}
m+l \\
t
\end{array}\right) a^{m+l-t} A_{m+l, t}
$$

$(\bmod p)$.

Thus for example, when $a=0,(5.9)$ yields

$$
p^{-h} S_{m} \equiv\left\{\begin{array}{ccc}
-(-1)^{k} & m & (l=1) \\
k ! & 2 & \\
(-1)^{k} & m(3 m+1) & (l=2) \\
k ! & 24 & \\
-(-1)^{k} & m^{2}(m+1) & (l=3) \\
k ! & 48 &
\end{array}\right.
$$

6. When $p=3$ it is easily proved that

$$
S_{m}(a)= \begin{cases}(-3)^{3 / 2}\left(\frac{2 m+a}{3}\right) & (m \text { even }) \\ (-3)^{(m-1) / 2} \hat{c} & (m \text { odd }),\end{cases}
$$

where $c=-2$ for $3 / m, c=+1$ for $3+m$. It is easily verified that (6.1) is in agreement with the general results above.

In conclusion a word may be said about the sum

$$
R_{m}(a)=\sum_{r=0}^{m}\left(\frac{r+a}{p}\right)\left(\begin{array}{c}
m \\
r
\end{array}\right) \text {. }
$$

If $m=m_{1} p+m_{0}, r=r_{1} p+r_{0}, 0 \leqq m_{0}<p, 0 \leqq r_{0}<p$,

then

$$
\left(\begin{array}{c}
m \\
r
\end{array}\right) \equiv\left(\begin{array}{c}
m_{1} \\
r_{1}
\end{array}\right)\left(\begin{array}{c}
m_{0} \\
r_{0}
\end{array}\right) \quad(\bmod p)
$$

so that (6.2) becomes

$$
R_{m}(a) \equiv \sum_{r}\left(\frac{r_{0}+a}{p}\right)\left(\begin{array}{c}
m_{1} \\
r_{1}
\end{array}\right)\left(\begin{array}{c}
m_{0} \\
r_{0}
\end{array}\right) \equiv 2^{m_{1}} R_{m_{0}}(a) . \quad(\bmod p)
$$

Thus to find the residue $(\bmod p)$ of $R_{m}(a)$ it suffices to consider the case $0 \leqq m<p$. However it is not evident how to find the residue in this case.

\section{REFERENCES}

[1] C. Jordan, Calculus of finite differences, second edition, New York, 1947.

[2] E. Landau, Vorlesungen über Zahlentheorie, vol. 1, Leipzig, 1927.

[3] T. Nagell, Introduction to number theory, New York, 1951.

[4] M. Ward, The representation of Stirling's numbers and Stirling's polynomials as sums of factorials, American Journal of Mathematics, 56 (1934), pp. 87-95.

\section{Duke University}

